\title{
Adverse reaction to dental local anaesthetic injection — 'allergy' is not the cause
}

\author{
J. P. Rood, ${ }^{\prime}$
}

Objective To investigate patients considered to have experienced allergic reactions to local anaesthetics administered for dental treatment.

Setting Dental facility within a general hospital.

Procedure Skin and intra-oral challenge tests.

Results No patients were found to be allergic to lignocaine or prilocaine. The most likely causes of the adverse reactions were found to be psychogenic.

Conclusion Although allergy to lignocaine (and the other amide anaesthetic agents used in dentistry) is known to be extremely rare, it continues to be suggested as a cause when adverse reactions to dental injections occur. Psychogenic reactions are the main causes of untoward events, and some can be alarming. A smaller proportion of adverse responses can be attributed to (avoidable) intravascular injections. Adverse reactions can be reduced if injections are administered carefully.

$\mathrm{T}$ The careful administration of local anaesthetic solution to facilitate dental treatment is an extremely safe procedure. Nevertheless, transient and trivial adverse reactions are common, ${ }^{1}$ but a few give rise to concern.

Allergic responses to the amide local anaesthetics used in dentistry are extremely rare. ${ }^{2,3}$ Many series of patients investigated for 'suspected allergy' have been reported, ${ }^{4-6}$ but in the vast majority of cases, hypersensitivity to the local anaesthetic agents was excluded. Exceptional cases have been reported in which an allergic response has been confirmed to one of the anaesthetics commonly used in dentistry (e.g. lignocaine, ${ }^{7}$ prilocaine ${ }^{8}$ or mepivacaine ${ }^{9}$ ) or another component of the dental product (eg methylparaben, ${ }^{10}$ metabisulphite ${ }^{11}$ ).

This report summarises the investigation of patients referred from dental colleagues for 'allergy testing' and the results reinforce the nature of adverse reactions which are commonly encountered.

\section{Method}

The first group of 97 patients was investigated over a 5-year period (1976-1981). The history of the adverse event was documented and patients informed that the testing procedure was designed to identify whether they had experienced an allergic reaction to the local anaesthetic agent used. The investigations were carried out in a recovery area in a general anaesthetic suite, with full resuscitation facilities and nursing supervision. Intra-dermal injections of plain lignocaine and normal saline (control) were performed on the first

$1^{*}$ Professor, Consultant in Oral and Maxillofacial Surgery, GKT Dental Institute, King's College Hospital, Caldecot Road, London SE5 9 RW

${ }^{*}$ Correspondence to: JP Rood

REFEREED PAPER

Received 27.10.99; Accepted 12.04.00

(C) British Dental Journal 2000; 189: 380-384
31 patients, then the testing procedure included plain prilocaine in the subsequent 66 .

Between 1982 and 1994, many patients were seen with similar problems, but details of their management were not retained. During this period, feed-back from practitioners revealed that negative results from skin tests alone did not convince patient or practitioner that the drugs were safe to use in dentistry. The testing procedure was therefore modified.

The following protocol (Fig.1) was adopted for the smaller recent group of 44 adult patients, investigated over 3 years (1994-96). This protocol is similar to that reported recently, ${ }^{12}$ but conducted in a dental environment.

\section{Preliminary arrangements}

On receipt of the referral, a mid-week appointment was arranged and the patient was informed that the visit would involve at least 3 hours at the hospital. Wherever possible, additional information was sought about the adverse event from the practitioner concerned.

\section{Preparation}

At consultation, a thorough history was obtained, to specifically include:

(a) The nature of the adverse event (this often proved the most informative aspect of the history).

(b) Previous dental care, including the use of local anaesthetics.

(c) Personal history of any events or disorders which could indicate an atopic background; associated family history.

(d) The origin of the suggestion that the patient might be allergic to local anaesthetics.

The testing process was explained and consent obtained. It was usually possible to inform the patient at this stage that, from the history alone, a true allergy could be excluded, so that the testing procedure would be comfortable and would provide useful information to help with their future management. A firm, confident approach was adopted to reassure the patient.

The testing procedure was arranged in a dental surgery and adjacent recovery area within a general hospital, which were equipped with emergency drugs and equipment. The procedures were conducted with the assistance of a trained nurse, who monitored the patient during the observation periods.

\section{The test procedure}

The patient was seated in the dental chair, which was then tilted so that the patient was semi-reclined.

Baseline recordings of the patient's pulse rate, blood pressure and oxygen saturation were obtained. The pulse oximeter remained in position during the tests. In cases where the history could not exclude an allergic reaction, venous access was secured.

The intra-dermal direct challenge tests were undertaken in the skin of the flexor surface of the forearm (which was cleaned using 


\section{Protocol for group of 44 patients}

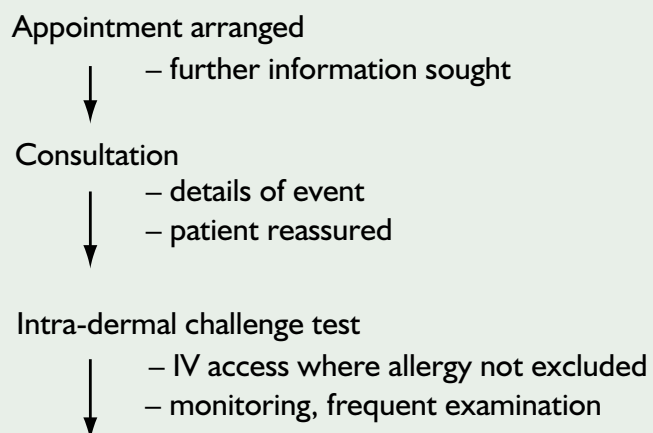

\section{Fig. I Recent protocol}

sterile saline). The test consisted of intra-dermal injections of $0.1 \mathrm{ml}$ of $0.5 \%$ lignocaine and $0.5 \%$ prilocaine (both plain without vasoconstrictor and without preservative), and normal saline (as a control). Although skin testing has been criticised (as false positive and negative reactions occur), they remain valuable as part of the testing procedure. ${ }^{13}$

The site of the injections and the patient's general condition were observed closely for the first 15 minutes, and then at 15 minute intervals up to 1 hour.

In the absence of an immediate response, the second stage of the procedure was conducted.

A 'dental' challenge test was undertaken. The dental chair was positioned so that the patient was fully reclined. $1.0 \mathrm{ml}$ of $2 \%$ lignocaine with adrenaline (Xylocaine, Astra) was administered as a maxillary buccal infiltration adjacent to the second premolar.

The patient was then monitored for a further hour, which included re-examination of the skin test sites.
At the completion of the observation period, the patient was discharged, but was requested to contact, or to return to hospital, on the following day (or later) if they had any concerns and, in particular, if they had any swelling, itch or a rash.

\section{Follow-up}

When the testing procedure was completed, a letter was sent to the dental practitioner (with a copy to any colleague who was involved with the referral) with a firm recommendation about the patient's management. A few months later, a further letter was sent to the dental practitioner, enquiring about the patient's subsequent management.

\section{Results}

\section{First group (Fig. 2)}

Of the initial series of 97 patients subjected to intra-dermal challenge tests, none was shown to be allergic to the anaesthetic agents, which always included lignocaine, and for most patients also involved prilocaine (no other agent had been implicated in the alleged allergic reactions). Twenty-two patients were considered to have suffered intravascular injections; the remaining 75 were thought to have experienced psychogenic reactions.

The symptoms exhibited by two patients involved sensory disturbances over distant contralateral areas of the body (limbs) after the administration of inferior alveolar nerve block injections. Both of these events were attributed to intravascular injections with cerebral distribution of the anaesthetic agent from the timescale of the responses, the absence of other signs (eg hyperventilation) and the failure to use an aspirating syringe for the injection.

During the skin testing procedure, one patient exemplified some of the problems of direct challenge testing by developing irritation at each of the injection sites. It was revealed that he had dermatographia (Fig. 3).

\section{Second group (Fig. 4)}

The second series of 44 patients were investigated, using the intradermal injection and a direct intra-oral challenge.

During this period, another nine were referred but not tested. The histories of seven patients so clearly excluded allergy to local anaesthetic agents that treatment was undertaken immediately using lignocaine with adrenaline as the local anaesthetic agent. Typically patients reported being regular dental patients and having received local anaesthetic injections on many previous occasions. The adverse event was described in a variety of ways, sometimes involving pain, dizziness, fainting or sickness. Two patients declined to be tested, one insisting that she was allergic and that she would seek treatment under general anaesthesia 'elsewhere'. The other patient declared that she suffered from multiple

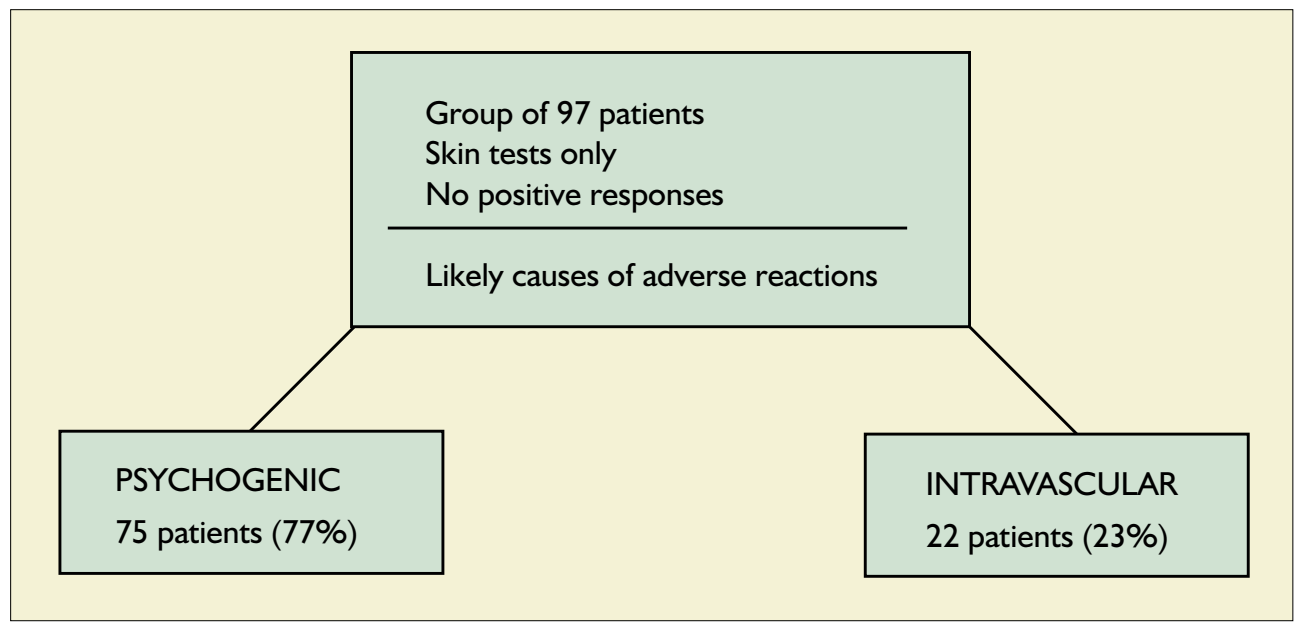




\section{RESEARCH anaesthesia}
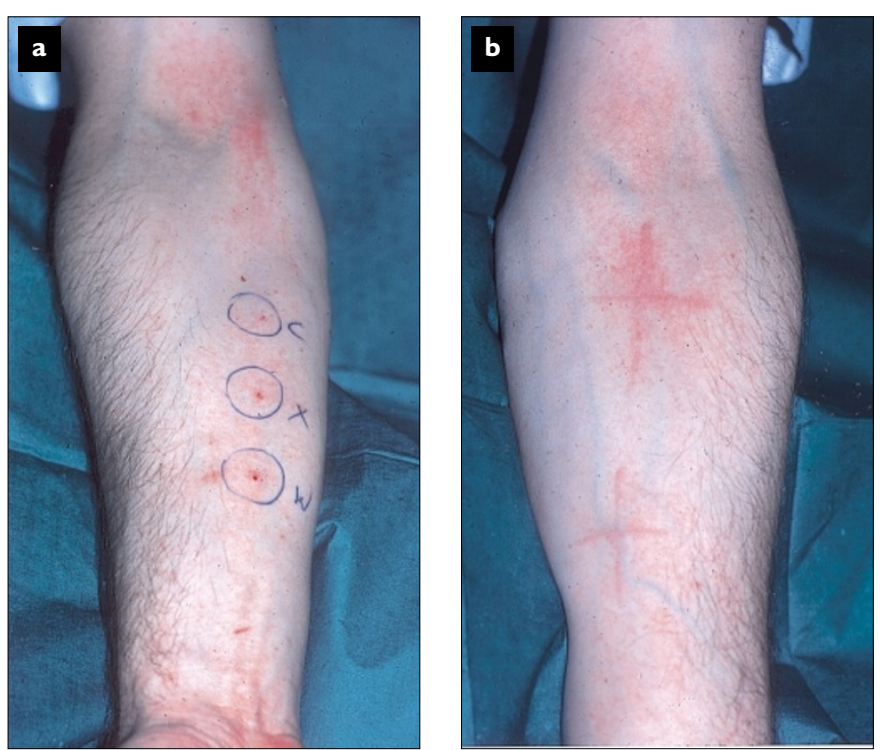

Fig. 3a Irritation at every skin injection site (false positive result) 3b Reaction to gentle stroking of skin with blunt probe 'dermatographia'

allergies and, at the first visit, stated that she must consult her (nonmedical) homeopathic practitioner. At a second visit, she explained that she had been advised not to undergo tests which would 'upset her system'.

Results from the 44 patients tested

Seven patients had been referred because they had informed their dental practitioner that they had suffered a previous allergic reaction. None of the histories supported that diagnosis and none proved to be allergic to the local anaesthetic agents.

One patient provided evidence that she had been tested and shown to be allergic to 'bisulphites' (her main adverse reactions being to foods containing sulphite preservatives). She had been informed that she was, therefore, allergic to all local anaesthetics. After satisfying the patient about the composition of various anaesthetics, the skin tests were undertaken and the patient was shown not to be allergic to lignocaine nor to the prilocaine solution. In this case, the dental challenge test was carried out with $1.0 \mathrm{ml}$ of $3 \%$
Citanest with Octapressin (Astra) without provoking an untoward response. It was recommended that solutions with adrenaline (containing metabisulphite) were avoided.

Three patients had been referred because the dental practitioner had observed localised swelling several hours following treatment - and one patient also developed a rash. One history indicated that there had been a bleed and the swelling was a haematoma, and the allergy tests proved negative. The other two could have been allergic in nature, but neither patient tested positive to local anaesthetics. Both patients had received restorative treatment, so had been exposed to a variety of materials - and one had returned to their practitioner for further treatment without local anaesthesia, after the referral but before testing and had again developed localised swelling. In these cases, it was recommended that the patients were referred to another specialist to identify the provocative material.

All of the remaining patients (33) were referred because of 'collapse' after receiving a local anaesthetic injection. The details of two patients are interesting:

\section{Case 1}

One patient had experienced her adverse reaction while receiving orthopaedic treatment, after which she was told that the cause was an 'allergy'. Several injections of bupivacaine had been administered to facilitate the manipulation of a painful shoulder. When attempts were made to treat the condition, pain was experienced, so several more injections were administered. A short while after the second battery of injections, the patient felt dizzy and may have lost consciousness briefly. Oxygen was administered and she slowly recovered. Bupivacaine was included as an additional local anaesthetic in the test procedure, without a positive response to any agent. The adverse reaction experienced by this patient was most likely to have been because of systemic toxicity.

\section{Case 2}

The description of another patient's adverse reaction was unusual. She had collapsed soon after receiving a dental local anaesthetic injection, with 'uncontrollable shaking' and respiratory distress. The referring dentist had witnessed the event and was concerned - an allergic reaction had been suggested as the possible cause. The skin tests were undertaken and at first the patient was comfortable. While under nursing supervision, about 10 minutes after

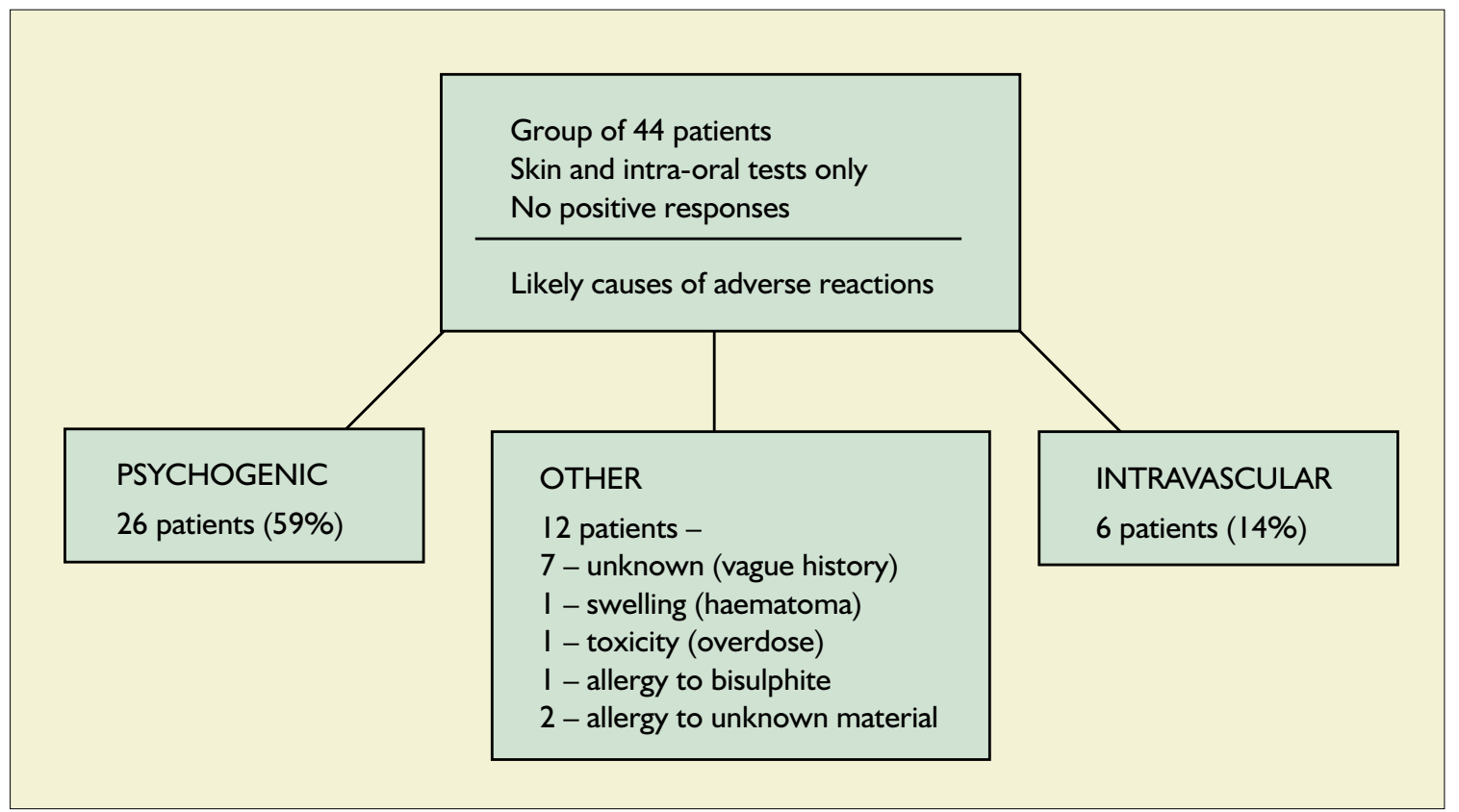


the intra-dermal injections had been completed, the patient began to experience her adverse reaction. This was a typical 'panic' attack with tachycardia (the pulse rate increased from 90 to 150), hyperventilation and tetany. There was a slight elevation of systolic blood pressure and the oxygen saturation remained constant. The patient was given reassurance and was encouraged to re-breathe. A similar case was recently reported ${ }^{14}$ and the mechanisms involved with this reaction have been described ${ }^{15}$ - less severe episodes are quite common.

The testing procedure was not continued on this occasion. The patient's husband confirmed that the reaction was typical of those experienced previously. The nature of the response was explained and accepted by the patient and her husband. The skin test was negative, so another visit was advised to undertake the direct dental challenge, but with the patient sedated. This was agreed.

At the second visit, the patient announced that, on reflection, she did not agree with the explanation given. Eventually the second challenge test was undertaken under sedation (intravenous Midazolam) and the patient recovered naturally, without any adverse event until 50 minutes later when a similar panic attack occurred.

It was recommended that the patient required careful supportive management and initially pre-medication or sedation could be employed to ensure that treatment was completed. The patient's husband was given advice about appropriate responses when panic attacks occurred.

The histories of the other 31 patients suggested vasovagal reactions in 25 and probable intravascular administration in 6: none was allergic to either of the anaesthetic agents.

One of this group was referred with a history of collapse associated with 'fitting': this male patient had a profound faint during the skin testing procedure and convulsions were avoided by promptly tilting the chair, so that the patient's feet were raised. The second dental challenge was administered uneventfully, with the patient fully reclined.

\section{Follow-up}

Thirty-two practitioners were contacted to enquire about the further management of their patients and 28 replied. Seven patients had not returned to their dental practitioner, although two had made appointments, which they did not keep. Six patients had not required a local anaesthetic injection. Fourteen patients had been successfully treated using local anaesthesia, although three continued to experience distress which the dental surgeons replying acknowledged were psychogenic reactions. In one case, it would appear that letters were not delivered: the patient received treatment at hospital under local anaesthesia without complication.

\section{Discussion}

Recently Wildsmith and colleagues ${ }^{12}$ reported the investigation of 25 patients, who were initially diagnosed as being allergic to local anaesthetic drugs: most of those patients ${ }^{15}$ had received dental treatment. Six of the dental patients were diagnosed as suffering from phobia, panic or anxiety and one patient had received an intravascular injection (being affected by the adrenaline content of the cartridge). One of the patients in that series was shown to be allergic to metabisulphite. Latex allergy accounted for problems with three patients - and the relevance of latex allergy to dental local anaesthesia has recently been drawn to the attention of the profession. ${ }^{16}$

Adverse reactions to dental local anaesthesia are common, but most are transient. ${ }^{1}$ Mild systemic reactions are also common being caused by intravascular injections or rapid absorption if excessive amounts of anaesthetic are administered. ${ }^{17}$ The more significant reactions, which cause concern, are primarily related to some form of 'collapse'. The results of investigations confirm previous reports that the vast majority of systemic reactions to dental injections are caused by:

\section{- Psychomotor responses (eg vasovagal or hyperventilation), and - Intravascular injections.}

The most common aspect of dentistry to provoke anxiety is an injection, ${ }^{18}$ so dental surgeons must be familiar with the variable signs and symptoms of psychological reactions to their treatment. Prompt management will prevent the development of additional worrying signs (eg tetany following hyperventilation; convulsions following a profound faint). Aspiration must be undertaken to avoid intravascular injections. ${ }^{19}$

After an adverse reaction has occurred, an explanation is frequently requested. An allergic reaction to the local anaesthetic should not be suggested. The term 'allergic' is recognised by patients and is readily adopted as the explanation. Experience has shown that the outcome of testing reassures some patients, but many remain committed to the original suggestion (and repeat the 'diagnosis' to the next practitioner they consult, resulting in another referral and reinforcement of their belief). Local anaesthesia is the mainstay of pain control during dental treatment, ${ }^{20}$ so that an adverse reaction must not be misdiagnosed as caused by an allergy, thereafter denying the patient access to safe pain control. Despite the numerous publications demonstrating that allergy is rare, practitioners still appear to suggest this frequently as a cause for untoward events.

When signs and symptoms develop which are genuinely suggestive of an allergic response, it is more likely to have resulted from contact with more common allergens (eg latex), so again the local anaesthetic should not be suggested as the probable cause. Where doubt exists or where the patient gives a 'definite history' (see earlier), it is important to refer the patient, but to inform them that the tests will be undertaken to confirm the safety of local anaesthesia and, if possible, to identify the true cause of the reaction. In the very rare cases where a patient is found to be allergic to one of the anaesthetic agents or to one of the other contents of a commercial preparation, a suitable alternative can usually be identified.

Most adverse reactions to local anaesthetic injections can be avoided if the following procedures are adopted:

- Confident, caring management; (for extremely anxious patients, pre-medication or sedation may be required)

- Supine positioning of patients (this will prevent the majority of faints)

- Aspiration (to avoid intravascular injections)

- Using preparations which do not contain methylparaben

- Slow injections (reduce discomfort and improve localisation of solution)

- Restricting total dose (for example, do not exceed $300 \mathrm{mg}$ of lignocaine - with or without adrenaline - for a healthy adult male of $70 \mathrm{Kg}$, ie 7 dental $2.2 \mathrm{ml}$ cartridges).

\section{Conclusions}

One hundred and forty-one adult patients have been investigated because 'allergy' was suggested to be the cause of adverse reactions which occurred during or immediately after dental local anaesthetic injections. In most cases the history excluded an allergic reaction. In all cases a direct skin (intra-dermal) challenge test, and additionally in 44 patients an intra-oral (typical dental) challenge test, failed to elicit an allergic reaction.

Adverse reactions to dental local anaesthetic injections are common, but the majority are transient and may go unnoticed by the dental surgeon. The most frequent causes of significant reactions 


\section{RESEARCH \\ anaesthesia}

are psychogenic (driven by anxiety). A smaller number of reactions which cause concern are caused by intravascular injections. Some patients suffer systemic reactions when larger amounts of solution are absorbed into the circulation.

When an adverse event occurs as a result of a dental local anaesthetic injection, the true nature of the problem should be considered carefully. It is important NOT to suggest that an allergic response has occurred when the clinical events are consistent with well-recognised common causes of adverse reactions to dental injections. Most adverse reactions are avoidable with attention to technique.

Allergy to lignocaine and the other amide local anaesthetics used in dentistry is extremely rare. If an acute reaction is strongly suggestive of an allergic response, early referral for thorough investigation is required.

1 Sadove M S, Wyant G M, Gittleson L A, Kretchmer H E. Classification and management of reactions to local anesthetic agents. J Am Med Assoc 1952; 148: $17-22$.

2 Aldrete J A. Sensitivity to lignocaine. Anaesth Intens Care 1979; 7: 73-74.

3 Brown D T, Beamish D, Wildsmith J A W. Allergic reaction to an amide local anaesthetic. Br J Anaesth 1982; 54: 435-437.

4 Incaudo G, Schatz M, Patterson R, Rosenberg M, Yamamoto F, Hamburger $\mathrm{R} N$. Administration of local anesthetics to patients with a history of prior adverse reaction. J Allergy Clin Immunol 1978; 61:339-345.

5 De Shazo R D, Nelson H S. An approach to the patient with a history of local anesthetic hypersensitivity: Experience with 90 patients. J Allergy Clin Immunol 1979; 63: 387-394.
6 Barer M R, McAllen M K. Hypersensitivity to local anaesthetics: a direct challenge test with lignocaine for definitive diagnosis. Br Med J 1982; 284: 1229-1230.

7 Noble D S, Pierce G F M. Allergy to lignocaine. A case history. Lancet 1961; 281: 1436.

8 Bateman P B. Multiple allergy to local anesthetics including prilocaine. J Med Aust 1974; 2: 449-452.

9 Johnson W T, De Stigter T. Hypersensitivity to procaine, tetracaine, mepivacaine and methylparaben: report of a case. J Am Dent Assoc 1983; 106: 53-56.

10 Luebke N H, Walker J A. Discussion of sensitivity to preservatives in anesthetics. J Am Dent Assoc 1978; 97: 656-657.

11 Schwartz H J, Sher T H. Bisulphite sensitivity manifesting as allergy to local dental anaesthesia. J Allergy Clin Immunol 1985; 75: 525-527.

12 Wildsmith J A W, Mason A, McKinnon R P, Rae S M. Alleged allergy to local anaesthetic drugs. Br Dent J 1998; 184: 507-510.

13 Aldrete J A, O'Higgins J W. Evaluation of patients with history of allergy to local anesthetic drugs. Southern Med J 1971; 64: 1118-1121.

14 Ezsias A. Lignocaine and anaesthetic allergy. Br Dent J 1998; 185: 428.

15 Spiers R L, Barsby M J. Hyperventilation in the dental chair. Dent Update 1995; 22: 95-98.

16 Field E A, Davies M, Longman L P, White J F. Latex allergy. Br Dent J 1997; 182: 410.

17 Rood J P. LA - Safe working dose. BDA National Dental Conference. April 1999.

18 Fiset L, Milgrom P, Weinstein P, Getz T, Glassman P. Psychophysiological responses to dental injections. J Am Dent Assoc 1985; 111: 578-583.

19 Meechan J G, Rood J P. Aspiration in dental local anaesthesia. Br Den J 1992; 172: 40.

20 General Dental Council. Maintaining Standards. May 1999. 